Published in final edited form as:

Pain. 2016 October ; 157(10): 2375-2381. doi:10.1097/j.pain.0000000000000660.

\title{
Impaired conditioned pain modulation in youth with functional abdominal pain
}

\author{
Matthew C. Morris, PhD ${ }^{1,2,3}$, Lynn S. Walker, PhD ${ }^{4,5}$, Stephen Bruehl, PhD ${ }^{5,6}$, Amanda L. \\ Stone, $\mathbf{M S}^{7}$, Alyssa S. Mielock, BA ${ }^{1}$, and Uma Rao, MD $^{4,5,8}$ \\ ${ }^{1}$ Department of Family and Community Medicine, Meharry Medical College, Nashville, TN \\ ${ }^{2}$ Center for Molecular and Behavioral Neuroscience, Meharry Medical College, Nashville, TN \\ ${ }^{3}$ Department of Psychology, Vanderbilt University, Nashville TN \\ ${ }^{4}$ Department of Pediatrics, Vanderbilt University School of Medicine, Nashville, TN \\ ${ }^{5}$ Vanderbilt Kennedy Center, Nashville, TN \\ ${ }^{6}$ Department of Anesthesiology, Vanderbilt University School of Medicine, Nashville, TN \\ ${ }^{7}$ Department of Psychology and Human Development, Vanderbilt University, Nashville, TN \\ ${ }^{8}$ Center for Behavioral Health Research, University of Tennessee, Knoxville, TN
}

\begin{abstract}
Functional abdominal pain (FAP) is associated with enhanced pain responsiveness. Although impaired conditioned pain modulation (CPM) characterizes adults with a variety of chronic pain conditions, relatively little is known about CPM in youth with FAP. The present study assessed CPM to evoked thermal pain in 140 youth (ages 10 to 17), 63 of whom had FAP and 77 of whom were healthy controls. Multilevel models demonstrated weaker CPM effects in FAP than healthy youth, as evident in slower within-person decreases in pain ratings during the conditioning phase. Weaker CPM effects were associated with greater somatic symptom severity and functional disability. Pain responses in FAP youth were heterogeneous, with $43 \%$ of youth showing an unexpected increase in pain ratings during the conditioning phase, suggesting sensitization rather than CPM-related pain inhibition. These findings highlight directions for future research on the emergence and maintenance of FAP in youth.
\end{abstract}

\section{Keywords}

functional abdominal pain; FAP; CPM; DNIC; adolescents

\begin{abstract}
Functional abdominal pain (FAP) refers to either episodic or continuous abdominal pain that remains medically unexplained despite thorough physical examination [36]. According to a recent meta-analysis, FAP is common in youth, with a worldwide pooled prevalence rate of $13.5 \%$ [18]. Many youth with FAP either meet current Rome III criteria for functional
\end{abstract}

\footnotetext{
Corresponding Author: Uma Rao, M.D., Address: University of Tennessee, CMHSRC, 122 Henson Hall, 1618 West Cumberland Avenue, Knoxville, TN 37996-3332, Phone: (865) 974-0840, FAX: (865) 974-1662, urao@utk.edu.
} 
gastrointestinal disorders, including irritable bowel syndrome (IBS), functional dyspepsia and abdominal migraine [2], or are at increased risk for developing functional gastrointestinal disorders over time [48]. FAP in youth is associated with significant functional disability, reflected in part by school absences [36], and is often persistent despite frequent utilization of health care services [13]. Although a variety of biological and psychosocial factors $[48,49]$ have been implicated in the development and maintenance of FAP in youth, little is known about the impact of altered pain modulatory systems.

Experimental pain responses, which may index functioning in pain modulatory systems, have proven useful as biomarkers of risk for chronic pain by discriminating clinical pain populations from healthy controls, correlating with changes in clinical pain, and predicting the development of chronic pain [11]. Pro-nociceptive pain modulation profiles are characterized by decreased inhibition and/or increased temporal summation to evoked pain [53]. One experimental method used to detect dysfunction in descending inhibitory pain modulatory systems is conditioned pain modulation (CPM; previously termed diffuse noxious inhibitory controls or DNIC), which refers to a reduction in perceived pain intensity for a test stimulus during application of a conditioning stimulus to a remote area of the body [44]. Impaired CPM predicts chronic post-operative pain when assessed in pain-free individuals prior to surgery [55], highlighting its potential utility as a biomarker of risk for chronic pain. Diminished CPM (relative to control samples) has been observed in a variety of chronic pain conditions, including fibromyalgia [19,22], temporomandibular disorder [17], and chronic headache [32]. Impaired CPM has also been found in adults with IBS $[14,17,31,51]$ and is linked to greater distress and discomfort in these individuals [15].

Few evoked pain studies have been conducted in healthy youth $[4,26,28,34]$ and only two have examined CPM [29,42]. Relatively little is known about experimental pain responses in youth with FAP. Lower heat and pressure pain thresholds - but no differences in pain tolerance - were found in a large sample of adolescents with IBS compared to healthy controls [41]. Another study of school-age children found lower temporal summation to both thermal and mechanical stimuli in children with recurrent abdominal pain compared to healthy controls, but no group differences in abdominal pain sensitivity [57]. Finally, a recent study restricted to females found impaired CPM in girls with IBS $(n=22)$ compared to healthy girls $(n=21)$, with the IBS group showing no change in pain ratings after application of the conditioning stimulus [52]. The present study is the first, to our knowledge, to compare CPM effects between FAP and pain-free youth in a relatively large sample including both genders. Based on findings from predominantly adult samples, we hypothesized that FAP youth would exhibit impaired CPM compared to healthy controls.

\section{Method}

\section{Participants}

FAP Group-Participants included pediatric patients between 11 and 17 years of age who presented to the pediatric gastroenterology clinic at a large university-based children's hospital for their initial evaluation for abdominal pain. Pediatric patients were considered eligible for this study if they reported recurrent abdominal pain for at least the past two months and could read and write in English at or above sixth grade level. Exclusion criteria 
included: (1) presence of chronic disease (e.g., inflammatory bowel disease, diabetes); and (2) hospitalization within the month prior to study enrollment. FAP patients were recruited as part of a larger, questionnaire-based study and a subset elected to complete the laboratory pain testing $(n=68)$. Through an examination of medical records from the clinic evaluation, one patient was excluded due to a diagnosis of inflammatory bowel disease. Four youth asked to discontinue the laboratory pain testing protocol and did not complete the CPM procedures. Thus, the final FAP sample was comprised of 63 youth.

Healthy controls-Controls $(n=77)$ were between 10 and 17 years of age and were recruited from a primary care clinic at a large university-based children's hospital and from the university's research recruitment website. As for the FAP group, the participants were required to read and write in English at sixth grade level or above. Exclusion criteria were as follows: chronic pain (defined as daily clinical pain $\geq$ three months in duration), use of prescription opioid analgesics, use of antidepressant or antianxiety medications, and learning difficulties requiring full-time special education services.

Additional exclusion criteria for both groups included sunburn or painful dermatological conditions at the time of the laboratory assessment and pregnancy. Study procedures were approved by the Institutional Review Board. All subjects and their parents provided written informed assent and consent, respectively, prior to beginning study procedures.

\section{Measures}

Demographic Information-Participants provided information on age, sex and race by self-report. Only youth with FAP completed sections A and B of the Questionnaire on Pediatric Gastrointestinal Symptoms (QPGS-RIII) [47]. These sections of the QPGS-RIII assess pain-related functional gastrointestinal disorders, specifically IBS and functional dyspepsia.

Somatic symptoms-The Children's Somatization Inventory - revised form (CSI) [45] was used to determine the perceived severity of somatic symptoms (e.g., headache, dizziness, nausea, back pain) in the past two weeks. All participants reported how much they were bothered by 24 somatic symptoms on a 5-point scale ranging from "not at all" (0) to "a whole lot" (4). Items were summed and total scores ranged between 0 and 96 (higher scores indicate greater perceived somatic symptom severity). In this sample, coefficient alpha for the CSI was .93.

Functional disability-The Functional Disability Inventory (FDI) $[7,46]$ was used to determine the perceived impact of general physical health on psychosocial and physical functioning. All participants reported the degree of difficulty they would have performing 10 specific activities due to their physical health on a 5-point scale ranging from 0 to 4 . Items were summed and total scores ranged between 0 and 60 (higher scores indicate greater functional disability). In this sample, coefficient alpha for the FDI was .92. 


\section{Evoked Pain Testing Procedures}

The CPM procedure is described in Figure 1. FAP youth were instructed not to take any pain medications within 4 hours of their laboratory visit. The 'test stimulus' for the CPM thermal pain protocol was a thermal pain stimulus delivered by a thermode $(30 \times 30 \mathrm{~mm})$ applied to the ventral forearm of the participant's non-dominant arm and administered via a computerized Medoc TSA-II Neurosensory Analyzer using commercially available software (TPS-CoVAS version 3.19, Medoc Inc., Ramat Yishay, Israel). As in previously published work [29], the 'conditioning stimulus' for the CPM protocol was a Boekel General Purpose Water Bath (Boekel Scientific, Feasterville, PA) maintained at a steady temperature of $46.5^{\circ} \mathrm{C}$ in accordance with previously-established guidelines [55]. Perceived pain intensity was rated by participants on a 0 to 100 scale $(0=$ "no pain" and $100=$ "worst imaginable pain"). Participants were trained in the use of the perceived pain intensity rating scale at the beginning of the pain protocol by providing examples of different pain stimuli; their understanding of the scale was checked through sample questions and during orientation to the pain testing equipment.

For each participant, the thermode temperature eliciting a pain rating between 50 and 70 was determined first (hereafter referred to as "P-60"). To determine the P-60 temperature, the thermode was applied to the non-dominant ventral forearm in sequences of 15 second pulses at $45^{\circ} \mathrm{C}, 46{ }^{\circ} \mathrm{C}$, and $47^{\circ} \mathrm{C}$, and at additional lower or higher temperatures as warranted until the P-60 was identified; thus, participants varied in the number of trials required to determine the P-60 temperature. Then, the thermode was moved to a non-overlapping location on the non-dominant ventral forearm, and the pre-conditioning test period was begun. The forearm thermal test stimulus was applied at the P-60 temperature continuously for a 30-second period, with three pre-conditioning pain ratings obtained at 10 -second intervals. Next, participants took a 10-minute break from the pain testing protocol to complete height and weight measurements and to rest. After the break, participants began the conditioning portion of the protocol by immersing their dominant hand in the hot water bath (conditioning stimulus) for 60 seconds. During this immersion, participants provided three water bath pain ratings at 0,10 and 20 seconds. At 30 seconds, the forearm thermal test stimulus was again applied at the P-60 temperature continuously for a 30-second period, with three conditioning pain ratings obtained at 40, 50 and 60 seconds.

\section{Data Analytic Plan}

Group differences in mean conditioning pain ratings controlling for mean pre-conditioning pain ratings (i.e., baseline corrected change) were examined using analysis of covariance (ANCOVA) in SPSS 22 for Windows (SPSS Inc. Headquarters, Chicago, Illinois, USA). For this analyses, the assumption of homogeneity of regression slopes was met. Recent evidence of mean differences in CPM between girls with and without IBS $(d=0.71)$ suggests that a minimum of 53 adolescents per group would be necessary to detect CPM effects [52]. Based on evidence of individual differences in pain rating changes across conditioning trials during CPM procedures [29], we examined within-individual changes in pain ratings from the mean pre-conditioning rating across the three conditioning trials. A multilevel model (MLM) was specified using hierarchical linear models (HLM 6) [37] consisting of a within-person (i.e., level-1) sub-model describing how each individual's pain ratings changed over successive 
trials, and a between-person (i.e., level-2) sub-model describing how these changes varied across individuals [5,39].

The Level 1 model was as follows:

$$
\text { Pain }_{t i}=\pi_{0}+\pi_{1} \text { Trial }_{t i}+e
$$

The Level 2 model was as follows:

$$
\begin{gathered}
\pi_{0}=\beta_{00}+\beta_{01} \mathrm{FAP}+\beta_{02} \text { Race }+\beta_{03} \mathrm{Sex}+\beta_{04} \text { Age }+r_{0} \\
\pi_{1}=\beta_{10}+\beta_{11} \mathrm{FAP}+\beta_{12} \text { Race }
\end{gathered}
$$

In this equation, Pain $_{\mathrm{ti}}$ indicates the numerical pain rating ( 0 to 100) at trial $t$ for person $i$, Trial denotes the pain rating (mean pre-conditioning, conditioning 1-3), and FAP denotes healthy controls (0) or functional abdominal pain (1). Of primary interest was the interaction between FAP and trial $\left(\beta_{11}\right)$. Significant interactions were probed, and simple slopes were calculated using Preacher and colleagues' online calculator. ${ }^{34}$ Demographic covariates (race, sex, age) were included as predictors of baseline pain ratings. The race $X$ trial interaction $\left(\beta_{12}\right)$ was controlled for, based on evidence of racial differences in CPM in healthy youth [29]. Preliminary analyses examined potential sex and age differences in CPM effects (i.e., the sex X trial and age X trial interactions) and tested whether CPM effects were associated with somatic symptom severity and/or functional disability. Normality assumptions were checked using a Q-Q plot of residuals. Exploratory analyses examined the distribution of CPM effects in FAP and healthy youth based on prior work suggesting significant variability in the strength and direction of these effects [52,56]. Three FAP youth were taking selective serotonin reuptake inhibitors at the time of their laboratory visit; removing them from analyses did not significantly alter any results.

\section{Results}

Descriptive statistics regarding demographic and pain responses for FAP and healthy youth are presented in Table 1. The groups did not differ significantly on age or sex. The FAP group included a higher percentage of Caucasians than healthy youth. Within the FAP group, a significant proportion met criteria for a pain-related functional gastrointestinal disorder: IBS $(n=39)$, functional dyspepsia $(n=4)$, IBS and functional dyspepsia $(n=3)$. Compared to the healthy group, the FAP group reported higher levels of somatic symptoms and functional disability.

Preliminary MLM analyses revealed a significant within-individual decline across the three pre-conditioning pain ratings ( $b=-4.0, S E=0.96, p<.001$ ); however, FAP and healthy youth did not differ in the rate of pre-conditioning decline ( $b=0.64, S E=1.44, p=.659)$. Both the somatic symptom severity (CSI score) $\mathrm{X}$ trial $(b=1.30, S E=0.53, p=.014)$ and functional disability $\mathrm{X}$ trial $(b=1.40, S E=0.53, p=.009)$ interactions were significant: individuals with weaker CPM effects reported greater somatic symptom severity and 
functional disability. Neither the age $\mathrm{X}$ trial $(b=0.08, S E=0.27, p=.782)$ nor the sex $\mathrm{X}$ trial $(b=-0.63, S E=1.04, p=.543)$ interactions were significant; that is, neither age nor sex influenced within-person changes in thermode pain ratings. Nevertheless, older youth had higher mean pre-conditioning thermode pain ratings $(b=2.54, S E=0.95, p=.009)$.

There were no significant group differences in P-60 temperature $\left(48.6^{\circ} \mathrm{C}\right.$ for both FAP and healthy youth), mean pre-conditioning pain rating, or in the first CPM trial rating. However, FAP youth reported significantly higher pain ratings in the second and third CPM trials compared to healthy youth. Although there were no significant group differences in the first and second water bath pain ratings, FAP youth reported significantly higher water bath pain ratings on the third trial. Consistent with lower CPM-related pain inhibition in the FAP group compared to healthy youth, ANCOVA showed that the FAP group had significantly higher mean conditioning thermode pain ratings after controlling for mean pre-conditioning thermode pain ratings $\left[F(1,129)=16.1, p<.001, \eta^{2}=.11\right]$.

\section{FAP and CPM effects}

MLM analyses examined whether FAP influenced within-person changes in thermode pain ratings from mean pre-conditioning rating across the three conditioning trials, controlling for the race X trial interaction. The FAP X trial interaction was significant $(b=3.69, S E=1.08$, $p=.001$ ) (Table 2, Figure 2). Simple slope analyses revealed that thermode pain ratings declined more slowly for FAP youth $(b=-4.19, S E=1.15, p<.001)$ than for healthy youth $(b=-7.79, S E=0.83, p<.001)$. Thus, CPM effects consistent with activation of descending inhibitory mechanisms were observed to some degree in both groups, but were elicited more strongly and rapidly for healthy youth. The race $\mathrm{X}$ trial interaction was significant $(b=2.30$, $S E=1.10, p=.037)$ and revealed weaker CPM effects in Caucasian compared to nonCaucasian youth.

\section{Distribution of CPM groups}

Exploratory analyses examined the distribution of CPM effects within FAP and healthy youth. Overall, pain inhibition and pain facilitation were differentially distributed between FAP and healthy youth $\left(\chi^{2}=8.56, p=.003\right)$. The percentage change from mean preconditioning to mean conditioning thermode pain ratings was $-19 \%$ in healthy youth (indicating pain inhibition) and $+4 \%$ in FAP youth (indicating pain facilitation) (Figure 3). The median CPM effect in the FAP group was $0 \%$, with 27 youth (43\%) showing mean thermode pain rating increases from the pre-conditioning to the conditioning phase. Higher mean thermode pain ratings during conditioning than during pre-conditioning were observed in a smaller proportion of healthy youth (19\%).

\section{Discussion}

Youth with FAP often exhibit functional impairment at school (absences, poor academic performance) and at home (less involvement in family activities) and are at increased risk for developing functional gastrointestinal disorders and psychiatric conditions $[1,36,48]$. The emergence of FAP is likely determined by complex interactions among social, environmental, psychological, biological and genetic factors [23]. The present study 
investigated one potential pathophysiological factor that has received relatively little attention in youth: altered function in pain modulatory systems. Specifically, we focused on $\mathrm{CPM}$, an evoked pain response measure believed to reflect descending pain inhibitory function. Results revealed impaired CPM in FAP youth of both genders compared to healthy youth, which complements evidence of impaired CPM in adults with IBS [14,17,31,51] and extends recent evidence of impaired CPM in girls with IBS [52]. Impaired CPM was associated with both greater somatic symptom severity and functional disability. Of note, individuals suffering from organic abdominal pain conditions such as Crohn's disease, oesophagitis or peptic ulcers exhibit intact CPM [3,9,27]. Thus, impaired CPM appears to accompany functional - but not organic - abdominal pain. These findings add to a growing literature highlighting the potential utility of impaired CPM as a shared vulnerability mechanism across a variety of chronic pain conditions [17,10,22,32].

The median CPM reduction in healthy youth was $19 \%$, which is similar to the $23 \%$ reduction reported by another study of healthy children and adolescents [42] but somewhat lower than the $29 \%$ reduction reported by a review of studies conducted in healthy adults [34]. These developmental differences are consistent with prior research suggesting that CPM effects strengthen from childhood to adolescence [42] but begin to weaken by middle age $[10,21,38,50]$. Strikingly, $43 \%$ of FAP youth showed a pro-nociceptive pattern - or pain facilitating pain - reflected in mean pain rating increases from the pre-conditioning to conditioning phase. Previous studies have described this counterintuitive pattern of results as either "pain facilitation" [52] or "less efficient CPM" [56]; the mechanisms that contribute to CPM-related sensitization require greater elucidation. Variability in both the strength and direction of CPM effects coupled with evidence of within-person changes in test stimulus pain ratings during the 30-second hot water bath immersion support the use of multilevel modeling over mean pain ratings derived from multiple test stimuli administrations. The latter can mask changes in CPM during the conditioning phase, which have previously been reported in healthy adults and adults with chronic fatigue syndrome using a variety of test and conditioning stimuli $[16,25,43]$.

CPM effects in the present study could not be explained by demographic factors or other features of evoked pain responses. Despite higher rates of many functional gastrointestinal disorders in females [6] and evidence of enhanced temporal summation to second pain (TSSP) in women with a history of FAP compared to men [8], we did not find evidence of sex differences in CPM nor have other studies conducted in healthy youth [42]. Racial differences in CPM have been found in youth [29] and adults [35]; CPM differences between FAP and healthy youth in the present study remained even after controlling for the race $\mathrm{X}$ trial interaction. The pattern of weaker CPM effects in FAP youth is remarkable given that FAP and healthy youth had virtually identical mean pre-conditioning pain ratings (58.2 and 59.8, respectively) and $\mathrm{P} 60$ temperatures $\left(46.8^{\circ} \mathrm{C}\right.$ and $46.8^{\circ} \mathrm{C}$, respectively). Prior work by our group has shown that a 'high pain dysfunctional' profile in pediatric FAP patients, defined in part by greater perceived pain threat, lower perceived coping efficacy, elevated pain catastrophizing and negative affect, was associated with enhanced temporal summation of pain at follow-up 9 years later [48]. Future studies should determine whether impaired CPM similarly distinguishes individuals with high pain dysfunctional profiles from other FAP patient subgroups, and if impaired CPM may even precede and predict FAP onset. 
Growing evidence of impaired CPM in chronic pain conditions has prompted investigation into the plasticity of pain modulation. Patients with osteoarthritis exhibit impaired CPM but show a normalization of pressure pain modulation following surgical pain relief [20]. CPM efficiency may influence treatment outcome: patients with painful diabetic neuropathy and impaired CPM report greater efficacy of duloxetine than patients with intact CPM and also show stronger CPM effects following treatment [56]. CPM effects could be mediated, in part, by endogenous opioid release: administering the opioid antagonist naloxone appears to dampen functional connectivity between the subgenual anterior cingulate cortex and regions implicated in descending pain control during a CPM task [40]. To our knowledge, no studies have yet examined whether psychotherapeutic interventions for FAP enhance CPM efficiency or if targeting impaired CPM can reduce risk for FAP onset.

Limitations of the present study provide directions for future research. First, we did not evaluate visceral sensitivity, which is more directly relevant to the clinical presentation of FAP in youth [49]. Examining relations between visceral sensitivity and CPM represents an important avenue for future research. Second, the pain testing protocol was only partially in line with recent recommendations for CPM assessment due to our reliance on one type of test stimulus [54]. Replication of findings across other test stimuli is critical given recent meta-analytic evidence that relations between psychosocial factors and CPM differ according to the pain modality [30]. Third, this study was cross-sectional; prospective studies are needed to determine whether impaired CPM is a risk factor for FAP. Fourth, we did not evaluate the impact of expectancies - which may influence CPM effects [12] - prior to the experimental protocol.

Despite these limitations, the current study adds FAP to a growing list of chronic pain conditions characterized by impaired endogenous pain inhibition [17,19,22,32]. That CPM effects - but not mean pre-conditioning pain ratings or P60 temperature - differentiated FAP from healthy youth provides further support for the importance of dynamic pain assessment modalities. Prospective evidence has shown that CPM efficiency - but not static pain assessments such as pain threshold - predict subsequent onset of chronic pain [55]. Youth with FAP exhibited heterogeneity in their responses to the CPM assessment and nearly half showed a counterintuitive increase in pain ratings during the conditioning phase; the mechanisms underlying this pattern of 'pain facilitating pain' should be investigated as well as its potential clinical and prognostic relevance. Understanding whether impaired pain inhibition influences the onset and course of FAP - and if CPM can be modified - is critical given the enormous toll of FAP on the individual and society.

\section{Acknowledgements}

Research was funded in part by grants from the National Institute of Health (UL1 RR024975/TR000445, G12 RR003032/MD007586, R01 MH068391, K01 MH101403, R01 DA017805, R01 DA031726, R01 DA037891, R01 HD76983, and P30 HD15052), by the Endowed Chair in Brain and Behavior Research at Meharry Medical College (Uma Rao) and by the Betsey R. Bush Endowed Professorship in Behavioral Health at the University of Tennessee (Uma Rao). These funding agencies had no further role in the study design, data collection, analysis or interpretation of data, writing of the report, or the decision to submit the manuscript for publication. The authors report no conflicts of interest. We gratefully acknowledge all individuals who participated in this study. 


\section{References}

[1]. Ammoury RF, Pfefferkorn MDR, Croffie JM. Functional gastrointestinal disorders: Past and present. World J Pediatr. 2009; 5:103-12. [PubMed: 19718531]

[2]. Baber KF, Anderson J, Puzanovova M, Walker LS. Rome II versus Rome III classification of functional gastrointestinal disorders in pediatric chronic abdominal pain. J Pediatr Gastroenterol Nutr. 2008; 47:299-02. [PubMed: 18728525]

[3]. Bernstein CN, Niazi N, Robert M, Mertz H, Kodner A, Munakata J, et al. Rectal afferent function in patients with inflammatory and functional intestinal disorders. PAIN. 1996; 66:151-61. [PubMed: 8880836]

[4]. Blankenburg M, Boekens H, Hechler T, Maier C, Krumova E, Scherens A, Magerl W, Aksu F, Zernikow B. Reference values for quantitative sensory testing in children and adolescents: Developmental and gender differences of somatosensory perception. PAIN. 2010; 149:76-88. [PubMed: 20138430]

[5]. Bryk, AS.; Raudenbush, SW. Applications and data analysis methods. Sage; Thousand Oaks: 1992. Hierarchical linear models.

[6]. Chang L, Toner BB, Fukuko S, Guthrie E, Locke GR, Norton NJ, Sperber AD. Gender, age, society, culture, and the patient's perspective in the functional gastrointestinal disorders. Gastroenterol. 2006; 130:1435-46.

[7]. Claar RL, Walker LS. Functional assessment of pediatric pain patients: Psychometric properties of the Functional Disability Inventory. PAIN. 2006; 121:77-84. [PubMed: 16480823]

[8]. Dengler-Crish CM, Bruehl S, Walker LS. Increased wind-up to heat pain in women with a history of functional abdominal pain. PAIN. 2011; 152:802-08. [PubMed: 21282006]

[9]. Drewes AM, Reddy H, Pedersen J, Funch-Jensen P, Gregersen G, Arendt-Nielsen L. Multimodal pain stimulations in patients with grade B oesophagitis. Gut. 2006; 55:926-32. [PubMed: 16091554]

[10]. Edwards RR, Fillingim RB, Ness TJ. Age-related differences in endogenous pain modulation: A comparison of DNIC in healthy older and younger adults. PAIN. 2003; 101:155-65. [PubMed: 12507710]

[11]. Edwards RR, Sarlani E, Wesselmann U, Fillingim RB. Quantitative assessment of experimental pain perception: Multiple domanins of clinical relevance. PAIN. 2005; 114:315-19. [PubMed: 15777856]

[12]. France CR, Burns JW, Gupta RK, Buvanendran A, Chont M, Schuster E, Orlowska D, Bruehl S. Expectancy Effects on Conditioned Pain Modulation Are Not Influenced by Naloxone or Morphine. Ann Behav Med. Jan 25.2016 [Epub ahead of print] PubMed PMID: 26809850.

[13]. Gieteling MJ, Bierma-Zeinstra SM, Passchier J, Berger MY. Prognosis of chronic or recurrent abdominal pain in children. J Pediatr Gastroenterol Nutr. 2008; 47:316-26. [PubMed: 18728528]

[14]. Heymen S, Maixner W, Whitehead WE, Klatzkin RR, Mechlin B, Light KC. Central processing of noxious somatic stimuli in patients with irritable bowel syndrome compared with healthy controls. Clin J Pain. 2010; 26:104-109. [PubMed: 20090435]

[15]. Jarrett ME, Shulman RJ, Cain KC, Deechakawan W, Smith LT, Richebé P, Eugenio M, Heitkemper MM. Conditioned pain modulation in women with irritable bowel syndrome. Biol Res Nurs. 2014; 16:368-77. [PubMed: 24463504]

[16]. Kakigi R. Diffuse noxious inhibitory control. Reappraisal by pain-related somatosensory evoked potentials following $\mathrm{CO}_{2}$ laser stimulation. J Neurol Sci. 1994; 125:198-05. [PubMed: 7807168]

[17]. King CD, Wong F, Currie T, Mauderli AP, Fillingim RB, Riley JL 3rd. Deficiency in endogenous modulation of prolonged heat pain in patients with irritable bowel syndrome and temporomandibular disorder. PAIN. 2009; 143:172-78. [PubMed: 19278784]

[18]. Korterink JJ, Diederen K, Benninga MA, Tabbers MM. Epidemiology of pediatric functional abdominal pain disorders: A meta-analysis. PLoS ONE. 2015; 10:e0126982. [PubMed: 25992621]

[19]. Kosek E, Hansson P. Modulatory influence on somatosensory perception from vibration and heterotopic noxious conditioning stimulation (HNCS) in fibromyalgia patients and healthy subjects. PAIN. 1997; 70:41-51. [PubMed: 9106808] 
[20]. Kosek E, Ordeberg G. Lack of pressure pain modulation by heterotopic noxious conditioning stimulation in patients with painful osteoarthritis before, but not following, surgical pain relief. PAIN. 2000; 88:69-78. [PubMed: 11098101]

[21]. Larivière M, Goffaux P, Marchand S, Julien N. Changes in pain perception and descending inhibitory controls start at middle age in healthy adults. Clin J Pain. 2007; 23:506-10. [PubMed: 17575490]

[22]. Lautenbacher S, Rollman GB. Possible deficiencies of pain modulation in fibromyalgia. Clin J Pain. 1997; 13:189-96. [PubMed: 9303250]

[23]. Levy RL, Olden KW, Naliboff BD, Bradley LA, Francisconi C, Drossman DA, Creed F. Psychosocial aspects of the functional gastrointestinal disorders. Gastroenterol. 2006; 130:144758.

[24]. Lu Q, Zeltzer L, Tsao J. Multiethnic differences in responses to laboratory pain stimuli among children. Health Psychol. 2013; 32:905-14. [PubMed: 23668844]

[25]. Meeus M, Nijs J, Van de Wauwer N, Toeback L, Truijen S. Diffuse noxious inhibitory control is delayed in chronic fatigue syndrome: An experimental study. PAIN. 2009; 139:439-48. [PubMed: 18617327]

[26]. Meier PM, Berde CB, DiCanzio J, Zurakowski D, Sethna NF. Thermal and vibration sensation and thermal pain detection thresholds in healthy children and adolescents. Muscle Nerve. 2001; 24:1339-45. [PubMed: 11562914]

[27]. Mertz H, Fullerton S, Naliboff B, Mayer EA. Symptoms and visceral perception in severe functional and organic dyspepsia. Gut. 1998; 42:814-22. [PubMed: 9691920]

[28]. Morris MC, Walker L, Bruehl S, Hellman N, Sherman AL, Rao U. Race effects on temporal summation to heat pain in youth. PAIN. 2015; 156:917-22. [PubMed: 25734994]

[29]. Morris MC, Walker L, Bruehl S, Hellman N, Sherman AL, Rao U. Race effects on conditioned pain modulation in youth. J Pain. 2015; 16:873-80. [PubMed: 26086899]

[30]. Nahman-Averbuch H, Nir RR, Sprecher E, Yarnitsky D. Psychological factors and conditioned pain modulation: A meta-analysis. Clin J Pain. Sep 3.2015 Epub ahead of print.

[31]. Piché M, Arsenault M, Poitras P, Rainville P, Bouin M. Widespread hypersensitivity is related to altered pain inhibition processes in irritable bowel syndrome. PAIN. 2010; 148:49-58. [PubMed: 19889500]

[32]. Pielsticker A, Haag G, Zaudig M, Lautenbacher S. Impairment of pain inhibtion in chronic tension-type headache. PAIN. 2005; 118:215-23. [PubMed: 16202520]

[33]. Preacher KJ, Curran PJ, Bauer DJ. Computational tools for probing interaction effects in multiple linear regression, multilevel modeling, and latent curve analysis. J Educ Behav Stat. 2006; 31:437-48.

[34]. Pud D, Granovsky Y, Yarnitsky D. The methodology of experimentally induced diffuse noxious inhibitory control (DNIC)-like effect in humans. PAIN. 2009; 144:16-19. [PubMed: 19359095]

[35]. Rahim-Williams FB, Rile JL. $3^{\text {rd }}$, Williams AK, Fillingim RB. A quantitative review of ethnic group differences in experimental pain response: Do biology, psychology, and culture matter? Pain Med. 2012; 13:522-40. [PubMed: 22390201]

[36]. Rasquin A, Di Lorenzo C, Forbes D, Guiraldes E, Hyams JS, Staiano A, Walker LS. Childhood functional gastrointestinal disorders: Child/Adolescent. Gastroenterology. 2006; 130:1527-37. [PubMed: 16678566]

[37]. Raudenbush, SW.; Bryk, A.; Cheong, YF.; Congdon, R.; du Toit, M. HLM 6: Hierarchical linear and nonlinear modeling. Scientific Software International; Lincoln, IL: 2004.

[38]. Riley JL 3rd, King CD, Wong F, Fillingim RB, Mauderli AP. Lack of endogenous modulation but delayed decay of prolonged heat pain in older adults. PAIN. 2010; 150:153-60. [PubMed: 20546997]

[39]. Singer, JD.; Willett, JB. Applied longitudinal data analysis: Modeling change and event occurrence. Oxford University Press; New York, NY: 2003.

[40]. Sprenger C, Bingel U, Büchel C. Treating pain with pain: Supraspinal mechanisms of endogenous analgesia elicited by heterotopic noxious conditioning stimulation. PAIN. 2011; 152:428-39. [PubMed: 21196078] 
[41]. Stabell N, Stubhaug A, Flægstad T, Mayer E, Naliboff BD, Nielsen CS. Widespread hyperalgesia in adolescents with symptoms of irritable bowel syndrome: Results from a large populationbased study. J Pain. 2014; 15:898-06. [PubMed: 24905280]

[42]. Tsao JCI, Seidman LC, Evans S, Lung KC, Zeltzer LK, Naliboff BD. Conditioned pain modulation in children and adolescents: Effects of sex and age. J Pain. 2013; 14:558-67. [PubMed: 23541066]

[43]. Tuveson B, Leffler A-S, Hansson P. Time dependant differences in pain sensitivity during unilateral ischemic pain provocation in healthy volunteers. Eur J Pain. 2006; 10:225-32. [PubMed: 15919219]

[44]. van Wijk G, Veldhuijzen DS. Perspective on diffuse noxious inhibitory controls as a model of endogenous pain modulation in clinical pain syndromes. J Pain. 2010; 11:408-19. [PubMed: 20075013]

[45]. Walker LS, Beck JE, Garber J, Lambert W. The Children's Somatization Inventory: Psychometric properties of the revised form (CSI-24). J Pediatr Psychol. 2009; 234:430-40. [PubMed: 18782857]

[46]. Walker LS, Greene JW. The Functional Disability Inventory: Measuring a neglected dimension of child health status. J Pediatr Psychol. 1991; 16:39-58. 1991. [PubMed: 1826329]

[47]. Walker LS, Lipani TA, Greene JW, Caines K, Stutts J, Polk DB, Caplan A, Rasquin-Weber A. Recurrent abdominal pain: Symptom subtypes based on the Rome II Criteria for pediatric functional gastrointestinal disorders. J Pediatr Gastroenterol Nutr. 2004; 38:187-91. [PubMed: 14734882]

[48]. Walker LS, Sherman AL, Bruehl S, Garber J, Smith CA. Functional abdominal pain patient subtypes in childhood predict functional gastrointestinal disorders with chronic pain and psychiatric comorbidities in adolescence and adulthood. PAIN. 2012; 153:1798-06. [PubMed: 22721910]

[49]. Walker LS, Williams SE, Smith CA, Garber J, Van Slyke DA, Lipani T, Greene JW, Mertz H, Naliboff BD. Validation of a symptom provocation test for laboratory studies of abdominal pain and discomfort in children and adolescents. J Pediatr Psychol. 2006; 31:703-13. [PubMed: 16861396]

[50]. Washington LL, Gibson SJ, Helme RD. Age-related differences in the endogenous analgesic response to repeated cold water immersion in human volunteers. PAIN. 2000; 89:89-96. [PubMed: 11113297]

[51]. Wilder-Smith CH, Schindler D, Lovblad K, Redmond SM, Nirkko A. Brain functional magnetic resonance imaging of rectal pain and activation of endogenous inhibitory mechanisms in irritable bowel syndrome patient subgroups and healthy controls. Gut. 2004; 53:1595-01. [PubMed: 15479679]

[52]. Williams AE, Heitkemper M, Self MM, Czyzewski DI, Shulman RJ. Endogenous inhibition of somatic pain is impaired in girls with irritable bowel syndrome compared with healthy girls. J Pain. 2013; 14:921-30. [PubMed: 23685184]

[53]. Yarnitsky D. Conditioned pain modulation (the diffuse noxious inhibitory control-like effect): Its relevance for acute and chronic pain states. Curr Opin Anaesthesiol. 2010; 23:611-15. [PubMed: 20543676]

[54]. Yarnitsky D, Boudhassira D, Drewes AM, Fillingim RB, Granot M, Hansson P, Landau R, Marchand S, Matre D, Nilsen KB, Stubhaug A, Treede RD, Wilder-Smith OHG. Recommendations on practice of conditioned pain modulation (CPM) testing. Eur J Pain. 2014; 14:339. [PubMed: 20227310]

[55]. Yarnitsky D, Crispel Y, Eisenberg E, Granovsky Y, Ben-Nun A, Sprecher E, Best LA, Granot M. Prediction of chronic post-operative pain: Pre-operative DNIC testing identifies patients at risk. PAIN. 2008; 138:22-28. [PubMed: 18079062]

[56]. Yarnitsky D, Granot M, Nahman-Averbuch H, Khamaisi M, Granovsky Y. Conditioned pain modulation predicts duloxetine efficacy in painful diabetic neuropathy. PAIN. 2012; 153:119398. [PubMed: 22480803]

[57]. Zohsel K, Hohmeister J, Flor J, Hermann C. Somatic pain sensitivity in children with recurrent abdominal pain. Am J Gastroenterol. 2008; 103:1517-23. [PubMed: 18510619] 
$\mathrm{CPM}=\%$ change in pain ratings for thermode during conditioning (hot water bath) compared to pre-conditioning (thermode only)

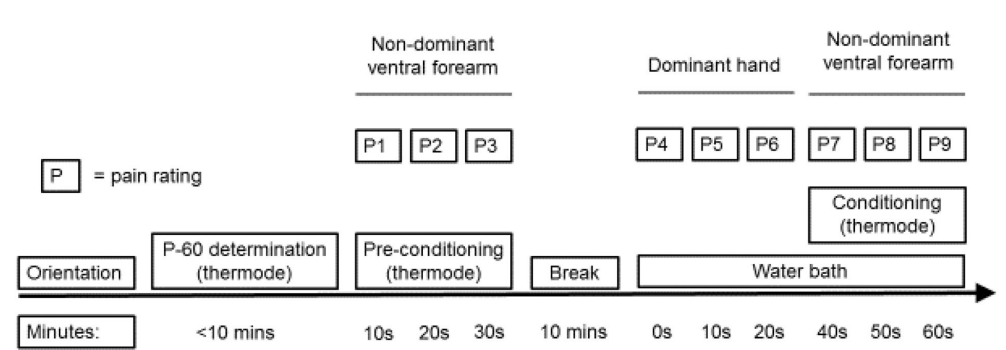

Figure 1.

Timeline for CPM procedure. 


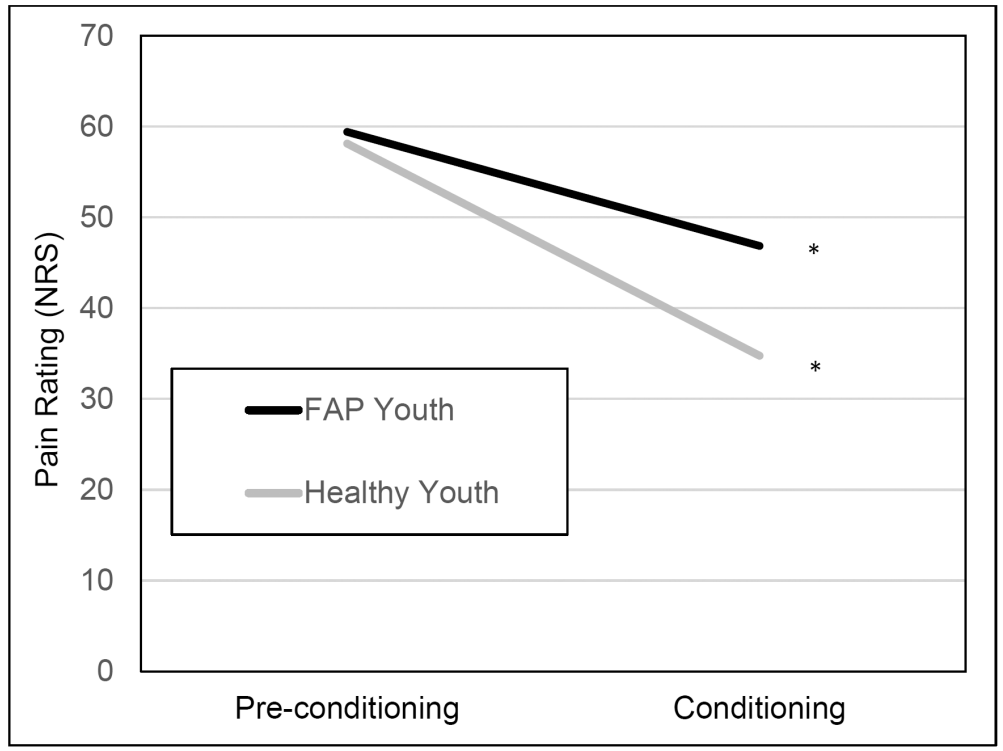

Figure 2.

Multilevel model testing the group (healthy control vs. FAP) by trial interaction as a predictor of test stimulus (i.e., thermode) pain ratings. $* p<.001$. 


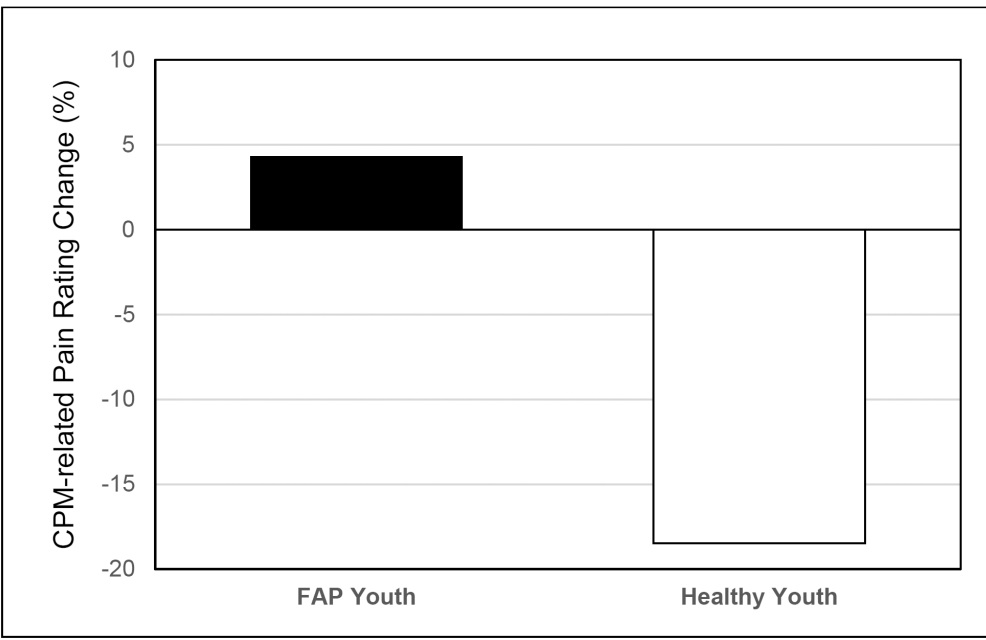

Figure 3.

Percentage change in mean pain ratings from the pre-conditioning to the conditioning phase in FAP and healthy youth. Note: negative values indicate pain inhibition and positive values indicate pain facilitation. 


\section{Table 1}

\section{Descriptive Data for FAP and Healthy Youth}

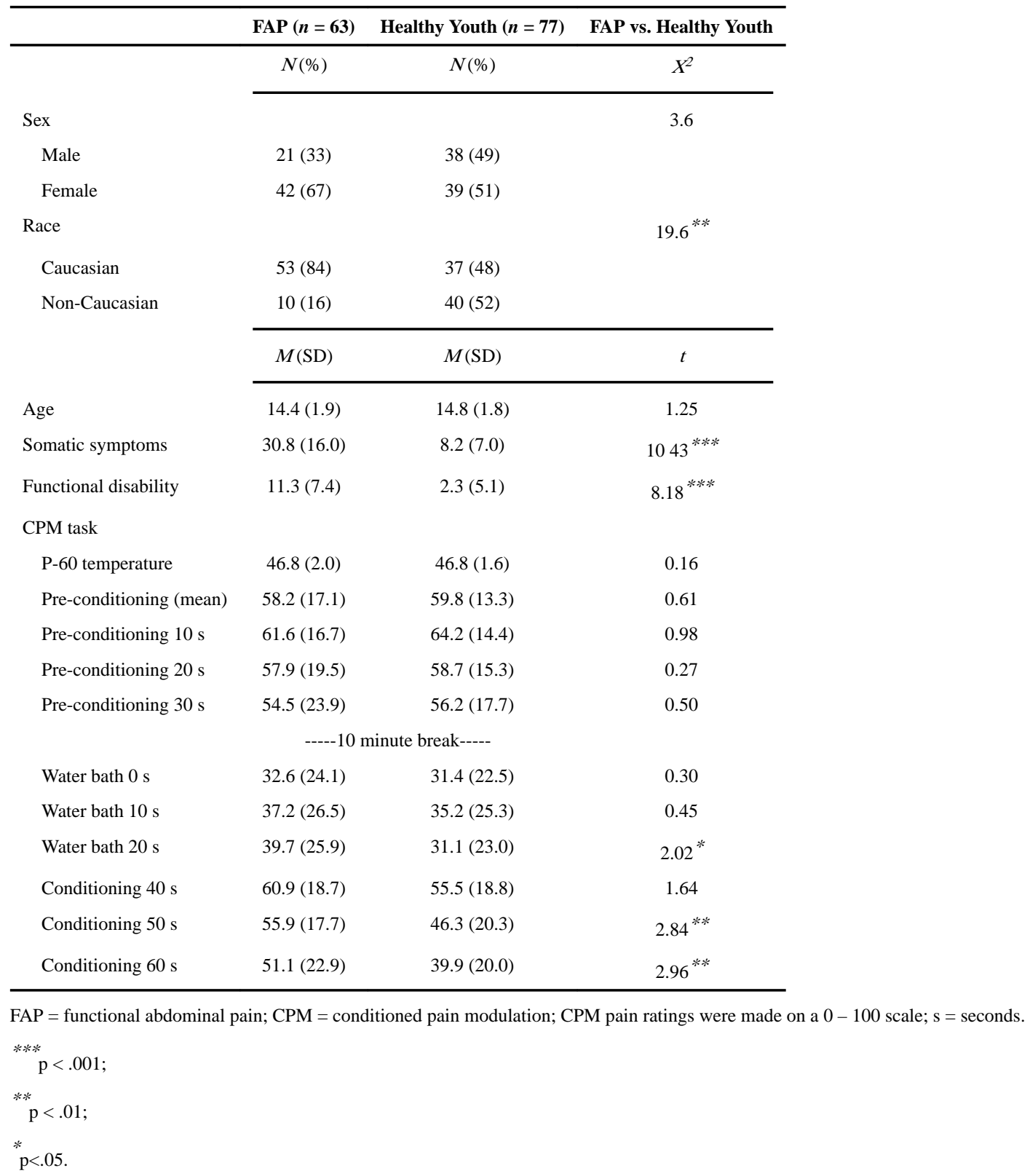

Pain. Author manuscript; available in PMC 2017 October 01. 


\section{Table 2}

Multilevel Model Predicting Changes in Pain Ratings Across Conditioning Trials

\begin{tabular}{lrrr}
\hline Predictor & Coefficient $(\boldsymbol{b})$ & Standard Error $(S \boldsymbol{E})$ & $\boldsymbol{p}$-value \\
\hline Intercept & 24.05 & 10.9 & .029 \\
Sex & 1.76 & 2.6 & .499 \\
Age & 2.99 & 0.7 & $<.001$ \\
Race & -4.10 & 3.9 & .291 \\
Group & -2.12 & 3.8 & .575 \\
Trial & -7.74 & 0.8 & $<.001$ \\
Race X Trial & 2.30 & 1.1 & .037 \\
Group X Trial & 3.69 & 1.1 & .001 \\
\hline
\end{tabular}

Group $(0=$ healthy control, $1=$ FAP $)$. 